\title{
Théâtralisation de la transmission dans Le Maître de Santiago de Montherlant
}

Dans Le Maître de Santiago (1947), pièce de théâtre écrite en 1945 (indication donnée par l'auteur à la fin du texte, p. 132), Henry de Montherlant situe l'action en Espagne en 1519. Le chevalier don Alvaro Dabo est considéré par ses pairs comme le chef spirituel de l'Ordre de Santiago, bien qu'en réalité celui-ci soit directement rattaché à l'autorité du roi. Lors d'une réunion mensuelle tenue chez le maître, cinq chevaliers appartenant à ce mouvement lui proposent de partir pour le Nouveau Monde pour faire fortune. Don Bernal est particulièrement intéressé par ce projet car son fils envisage d'épouser Mariana, la fille de don Alvaro. Ascète et hostile à toute forme de lucre, le père de Mariana rejette catégoriquement cette proposition. Cela engendre un conflit qui va faire émerger deux attitudes diamétralement opposées, avec, d'un côté, un vif désir de participer à l'évangélisation des Indes d'Amérique et de gagner beaucoup d'argent et, de l'autre, un rejet total de cette action et de son utilisation pour un enrichissement excessif et illicite. Cela offre à don Alvaro l'occasion d'exercer sa fonction de guide et de chercher à transmettre à ses destinataires les valeurs qu'il considère comme fondamentales. Face à son intransigeance, il ne reste plus à don Bernal qu'une seule possibilité : avec la complicité de Mariana, lui envoyer un faux émissaire du roi pour le contraindre à accepter le départ. Le procédé est près de réussir, ne serait la décision prise par la fille d'Alvaro de dévoiler la supercherie. Les rapports distants entre père et fille cèdent ainsi la place à une communion qui les déterminera à se retirer dans un couvent.

- Hamdi Hemaïdi - professeur de littératures française et francophone à la Faculté des Lettres, des Arts et des Humanités, Université de la Manouba (Tunisie), Campus Universitaire de la Manouba, 2010 La Manouba (Tunisie). Adresse de correspondance : hamdihemaidi@yahoo.fr

ORCID iD: https://orcid.org/0000-0002-8096-3290 
La question du rapport maître-disciple se pose dans cette pièce d'une façon un peu particulière. Ni don Alvaro, le protagoniste, ne se considère comme un enseignant, ni les personnages qui gravitent autour de lui ne se comportent en élèves. Et pourtant, par le biais d'une théâtralisation bien ficelée, nous nous orientons dans le schéma complexe de la transmission didactique telle qu'elle apparaît dans ses diverses formes et aussi bien au niveau de l'énoncé (discours des personnages) qu’à celui de l'énonciation (discours de l'auteur). Plusieurs destinateurs s'adressent dans cette pièce à plusieurs destinataires, avec parfois des émetteurs qui sont également des récepteurs et vice-versa. Nous nous proposons ici d'étudier les différents types de rapports qui se tissent entre les uns et les autres.

La théâtralisation de la transmission fait parler deux types de voix : la voix des êtres de papier ou personnages et celle de l'auteur qui les a créés (Montherlant). Il convient donc de commencer par l'examen des différents aspects de cette théâtralisation. En tant que texte dramatique, Le Maître de Santiago est, grâce au dialogue, un cadre idéal pour l'échange et, par là-même, pour la circulation des idées. Sur les quinze scènes qui constituent les trois actes de la pièce, seules deux sont des monologues (I 7 et III 2). Les scènes à deux personnages, qui correspondent à la situation idéale pour l'exercice d'une influence, sont au nombre de huit et, de ce fait, les plus fréquentes. Bien que refusant d'agir, don Alvaro est érigé en protagoniste et mis en évidence par sa forte présence (apparition dans onze scènes sur quinze). De plus, Montherlant exploite l'illusion dramatique (dans sa double acception de « fiction» et de « jeu ») en montrant des personnages qui font semblant de ne pas s'inscrire dans la relation maître-disciple alors qu'ils y adhèrent totalement. D’autre part, l'auteur définit luimême Le Maître de Santiago comme un " auto sacramental » (Montherlant, 1991, p. 136). Ce genre de théâtre consiste en une allégorie religieuse qui, dans sa version classique, prend la forme d'une psychomachie (vertus $v s$ vices). Ici, le conflit est, selon Montherlant, axé sur les "démêlés de l'homme et de la Grâce " (Montherlant, 1991, p. 139) et, par conséquent, sur l'initiation aux épreuves conduisant au salut. À cela s'ajoute le fait que dans l'intrigue c'est le discours-action qui se substitue à l'action proprement dite : don Alvaro refuse de satisfaire la demande de ses compagnons de partir pour le Nouveau Monde.

Quant au mélange de la réalité et de la fiction, il agit au profit d'un souci didactique et de vraisemblance qui renforce la plausibilité de la situation exposée. En effet, l'ancrage historique est assuré par le moyen de la date à laquelle se déroulent les faits (1519), du lieu où se passe l'action (Avila, ville de Castille) et de l'appartenance des chevaliers à l'Ordre de Santiago, qui a réellement existé. Mais il nous est signalé que si « don Alvaro a une forte vraisemblance historique », « dans l'intrigue [...] tout est fiction : il n'y a pas un emprunt» (Montherlant, 1991, p. 137).

L’oblicité de la transmission est également matérialisée par la polysémie du terme " maître » que comporte le titre : chef détenteur d'une autorité, propriétaire, enseignant, compétent sur le plan professionnel, dominateur... Cela est d'autant plus déroutant qu'en 1519 l'Ordre de Santiago a pratiquement cessé d'exister depuis qua- 
rante-cinq ans et que son grand maitre n'est autre que le roi d'Espagne. Par ailleurs, la théâtralité est inhérente à toute forme de transmission. Les théoriciens nous indiquent que les gestes y imitent ceux de l'amour. Il est naturel qu'elle se retrouve dans Le Maître de Santiago.

Nous adopterons dans l'analyse du fonctionnement de la transmission dans cette pièce une démarche thématique en essayant de rattacher ce que révèle le texte aux principales analyses théoriques portant sur cette question.

\section{Figures du maître}

Montherlant a centré l'intrigue sur les divergences qui déchirent cinq chevaliers de l'Ordre et leur « chef » don Alvaro Dabo au sujet du départ pour les Indes en vue de faire fortune, lors d'une réunion qui a lieu chez celui-ci. Les divergences prennent un caractère particulièrement orageux lorsque le conflit se focalise sur don Alvaro, père de Mariana, et don Bernal, père de Jacinto, dont les enfants s'aiment et projettent de se marier. Don Bernal estime que la réussite de cette union est tributaire de la fortune que don Alvaro irait amasser au Nouveau Monde, tandis que celui-ci est farouchement opposé à un projet de ce genre. Il saisit cette occasion pour sériger en éveilleur de consciences et développer un réquisitoire contre le comportement des Espagnols à l'égard des autochtones d'Amérique. Il est de ce fait la principale figure du maitre. D’autres figures apparaissent en filigrane (en particulier Dieu et Montherlant lui-même).

Quelles sont les particularités de cette figure principale ? Don Alvaro, c'est d'abord un nom propre motivé, parce que chargé de significations... antinomiques. $\mathrm{Si}$ « Alvaro » veut dire " noble » et réfère à l'origine sociale du personnage, « Dabo » comporte des acceptions multiples. La première relève du domaine religieux : « frère quêteur dans les couvents des ordres des mendiants ». La seconde équivaut à « dupe ». La troisième correspond à un emploi péjoratif: « souffre-douleur, niais». La quatrième est à mettre en relation avec le personnage lui-même : "Il se conforme à notre plus ancienne devise : Dedi et dabo, "J'ai donné et je donnerai" " (Montherlant, 1991, p. 23). Ces indications donnent du protagoniste une image problématique. Il est à la fois celui qui peut prêter à rire et celui qui réunit les traits distinctifs du maître : noblesse (d'âme), humilité (du quêteur) et générosité infinie. Montherlant semble de la sorte nous suggérer qu'il s'agit d'un être à deux facettes. D'ailleurs, il le confirme lui-même : " Je n'ai pas fait d'Alvaro un chrétien modèle, et il est par instants une contrefaçon du chrétien : presque un pharisien» (Montherlant, 1991, p. 137).

Âgé de quarante-sept ans, veuf et père d'une fille unique Mariana, don Alvaro "s'est retiré du métier des armes [...] après sêtre couvert de gloire à vingt ans " (Montherlant, 1991, p. 34). Le texte nous offre de lui l'image d'un être tridimensionnel : en tant qu'homme, en tant que géniteur et en tant que maître, il constitue un écart par rapport à la norme. 
Alvaro l'homme se manifeste à travers ce qu'il nous révèle de lui-même et ce que pensent de lui les autres personnages. Dans son auto-description, il nous dévoile ce qui, selon lui, fait la particularité d'un maitre : ce qu'il est, les paradoxes qu'il cultive, ce à quoi il aspire et ce qui constitue sa singularité. Il est à cheval sur les principes (voir p. 72). Il possède une culture que tout le monde n'a pas, ce qui transparaît à travers ses lectures : une chanson de geste allemande dont le héros est un chevalier de l'Ordre Teutonique (voir p. 30 et 33, ordre dont l'idéal est de servir les pauvres et de livrer la guerre aux Infidèles), les contes marocains qui contiennent une sagesse populaire qu'il approuve totalement, des romances tirés de chansons de gestes (voir p. 105 et 107) et qui présentent l'épreuve envoyée par Dieu comme " une marque de sa prédilection » (p. 105), le Parsifal de Wolfram d'Eschenbach défini comme étant «le Cantique des Cantiques de la chevalerie» (p. 107).

Cette personnalité forgée à partir de la chevalerie comme modèle l'autorise, selon lui, à se démarquer du commun des mortels. Il rejette la notoriété : "J'aime être méconnu » (p. 42). Il n’a « nulle ambition » (p. 41). Il hisse la pauvreté au rang de l'élection divine : «Dieu m'a fait cette grâce particulière de me rendre pauvre » (p. 79). Compte tenu de cette singularité, ce à quoi il aspire ne peut être qu'un cheminement en dehors des sentiers battus. Il exprime graduellement dans ses propos la quête d'un véritable absolu. La «soif [...] d'un immense retirement» (p. 41) devient une faim « de silence et de solitude » (p. 67) avant de se transformer en " une abstraction complète » (p. 67) permettant de vivre « des journées vides, si vides...» (p. 67). Nous retrouvons ainsi un homme qui se dit posséder des qualités inégalables, qui se réclame de la « race de la rigueur» (p. 76) et qui « ne tolère que la perfection» (p. 81).

Les membres de son entourage complètent cet autoportrait, mais surtout tentent de rétablir une part d'objectivité dans l'évocation du personnage. Mettant l'accent sur la générosité extrême de son père, Mariana souligne que, loin d’être un égoïste, il a «horreur [...] de son intérêt » (p. 29) et va même jusqu’à affirmer qu' «il éprouve du plaisir à être dépouillé » (p. 22). Elle assure également que tout est naturel dans son comportement : "Il n'y a nulle affectation en mon père. Il va droit devant lui " (p. 93). Selon elle, sa visée ultime est « son salut propre» (p. 93). Nous retrouvons ici les caractéristiques du modèle à suivre, celle du maître exemplaire qui communique les valeurs à adopter par sa façon dêtre. Bernal ne voit pas les choses du même œil. D’après lui, Alvaro a les défauts de ses qualités. Son égoïsme est mentionné plus d'une fois : "Vous sacrifiez votre enfant à vous-même, à vous-même et rien qu'à vous-même » (p. 75), " vous choisissez toujours, en définitive, la voie qui vous plait » (p. 88). Il en résulte, d'après lui, un manque de sincérité : " se piquer de ne parler jamais argent est une fausse élégance » (p. 78). Il en découle également une tendance au paradoxe pour se démarquer des autres, « une pente à contredire» (p. 92) et, en fin de compte, une « cruauté » (p. 75) manifeste. De ce fait, la générosité n'est plus qu'un prétexte servant à justifier la démission et le refus de s'impliquer pour que l'image affichée ne soit pas égratignée : «Vous vous êtes réfugié dans la charité. S'il vous fallait agir, ce qui s'appelle agir, vous vous crotteriez comme les autres» (p. 76). Bernal 
nous propose un contreportrait qui fait d'Alvaro un être qui se nourrit d'utopie et qui n'est pas un exemple à suivre : "Vous êtes un de ces esprits charmés de leurs propres rêves, qui peuvent devenir si dangereux pour une société » (p. 77). Cette description est-elle pour autant à prendre pour argent comptant quand on sait que tous les griefs formulés ici sont en relation avec le refus d'Alvaro de partir pour le Nouveau Monde afin d'y faire fortune et de permettre à Jacinto d'épouser la fille d'un homme riche ? Il en est de même pour cette assertion du comte de Soria, qui vient d'être congédié par Alvaro : « les vieux, souvent, avec des apparences saintes, ont le cœur dur et orgueilleux » (p. 121).

Alvaro le géniteur se démarque-t-il d'Alvaro l'homme? Avant de se déterminer à prendre en charge l'initiation de sa fille et de jouer pleinement le rôle du maître, il a entretenu avec Mariana des rapports tensifs en progression constante. Bien que celle-ci ait toujours évité d'entrer en conflit avec lui, il n'a cherché à lui servir de guide qu'après une péripétie qui lui a révélé à quel point il avait tort.

Au départ, il a évité d'établir le contact : "Nous ne nous voyions qu'aux repas » (p. 69). Puis il a choisi l'indifférence : "Jeune fille, sa vie [...] ne m'intéressait pas » (p. 69). Il considère cela comme le meilleur moyen d'éviter le conflit : « je ne lui demande rien, ne lui reproche rien, nous ne nous heurtons jamais » (p. 71). Toutefois, les tensions sont vite apparues et se sont développées. La présence de la jeune fille a d'abord été ressentie comme importune : «Je l'entendais marcher, il arrivait qu'elle chantât... Elle me lassait souvent, et m’importunait à l'occasion » (p. 67). Par la suite, elle est devenue un fardeau : « il est lourd d'avoir une fille» (p. 67-68). Enfin, elle n'est plus que sa " pierre d'achoppement» (p. 87) et, de ce fait, elle le « dégradait» (p. 69). Rejetée, elle n’est plus désignée par ses qualifications humaines, elle n’est plus que « quelque chose qui n'existe que par un de mes instants de faiblesse » (p. 75).

Comment aborde-t-il la question de son mariage ? N'y voyant aucune opposition de principe, il estime que c'est plutôt un moyen de se débarrasser d'elle : " $\mathrm{Ma}$ maison n'est pas gaie [...] peut-être serai-je plus heureux, quand elle n'y sera plus » (p. 66-67). Toutefois, il n’est nullement disposé à s'en occuper : « Pour chercher à établir Mariana, il aurait fallu que je me perdisse. En soucis de société et en gaspillage de temps » (p. 66). Poussant son raisonnement plus loin, il exclut les relations affectives père-fille " afin de n'être pas enchaîné par la tendresse paternelle » (p. 77). Il remet en question son statut de père : " être le père d'une fille est-il être père ? » (p. 87). Plus encore, il condamne la relation familiale : « la famille par le sang est maudite » (p. 83). Il assimile l'existence des petits-enfants à une souillure : " pas d'enfants, personne pour me salir» (p. 128). C'est dans ce contexte que s'inscrit sa diatribe contre le sentiment amoureux. Il réitère plus d'une fois le mépris qu'il éprouve à son égard: "On me dit que vous avez pris je ne sais quel sentiment pour le fils de Bernal » (p. 86), «j'ai horreur de ce genre » (p. 86). Il ne l'apprécie pas : «Les attachements me déplaisent » (p. 66). Il le déprécie même : "Vous êtes une petite singesse, rien de plus. Et tout cet amour entre hommes et femmes est une singerie. Sachez que vous êtes enfoncée en pleine grimace, en plein ridicule, et en pleine imbécillité » (p. 86). 
Il le repousse parce qu'il le considère comme une maladie : «va, tu n'auras pas connu l'infection de l'amour du mâle » (p. 128). Il le condamne en tant que crime : « Dans les contes marocains, il y a un personnage classique : le père qui médite de faire tuer sa fille, parce qu'il la voit amoureuse » (p. 85).

Quel statut Alvaro a-t-il ? Pour Tia, la duègne, le véritable détenteur du pouvoir est quelqu'un d'autre : "on surnomme votre père "Le Maître de Santiago" » (p. 18). Précision confirmée par Mariana : « depuis vingt-cinq ans, c'est le Roi qui est le Grand Maitre des trois Ordres de chevalerie espagnols» (p. 18). Mais pour les membres de cette communauté, il s'agit d'autorité effective : « Don Alvaro, vous que nous nommons si respectueusement et si affectueusement "le Maître de Santiago" [...]» (p. 39), dit Olmeda, qui persiste et signe : « vous êtes bien le Maître de Santiago » (p. 57).

Doté de ce pouvoir, le père de Mariana n'hésite pas à se comporter en tant que chef et en tant que guide. Il pratique la rigueur quand il organise des réunions : il «persiste à $[\ldots]$ offrir une hospitalité $[\ldots]$ austère » (p. 19) et, de ce fait, il ne sert à ses convives que de l'eau, parce qu'elle est « le symbole de la pureté » (p. 20) et parce qu'il « trouve qu'il est indécent que des sujets d'une certaine gravité soient mêlés à des soucis de nourriture » (p. 19). Qui plus est, il vit et fait vivre sa fille dans le dénuement total : " Pour mon père, seul est important, ou plutôt seul est essentiel, ou plutôt seul est réel ce qui se passe à l'intérieur de l'âme " (p. 22). Cette situation l'autorise également à définir la ligne de conduite que sa communauté doit absolument respecter : "Quelque déchu qu'il soit, l'Ordre est le reliquaire de tout ce qui reste encore de magnanimité et d'honnêteté en Espagne. Si vous ne croyez pas à cela, démettez-vous-en. Si nous ne sommes pas les meilleurs, nous n’avons pas de raison d’être " (p. 52). Mais ce statut semble ne pas le satisfaire entièrement, parce que peu conforme à sa véritable quête, parce qu'il le dote de peu de moyens pour atteindre ses objectifs : «Il y a un Ordre du Silence : de celui-là aussi je devrais être Grand Maître » (p. 50-51). Paradoxalement, la recherche de cette nouvelle autorité n'est que l'expression litotique d'une démission, d'un retrait annoncé puis accompli à la fin de la pièce. Le silence est ici synonyme de mort symbolique.

Au père de Mariana s'ajoutent deux autres figures du maître. L'Ordre de Santiago suit la règle de Saint-Augustin. Selon celui-ci, « Nous parlons, mais c'est Dieu qui enseigne » (cité par Franck, 2003). Dieu est donc une figure magistrale dans cette pièce. Présente-absente, elle intervient sans qu'on la voie sur scène. Ce sont ses disciples qui nous parlent d'elle. Pour eux, elle est surtout volonté.

En tant que troisième figure du maitre, le Roi brille par son absence.

En plus des personnages remplissant la fonction magistrale, se profile à travers la pièce l'image d'un auteur (aux sens multiples du terme : autorité, créateur, modèle à imiter, guide, conseiller, protecteur) qui s'emploie à communiquer une sagesse à ses lecteurs-spectateurs. Les points de rencontre et de divergence entre Montherlant et son protagoniste se lisent dans sa pratique scripturale de valorisation/dépréciation, qui invite à déceler un message émis à l'intention des récepteurs de l'œuvre par un écrivain qui se veut engagé en faveur du processus de décolonisation. 
Les différentes figures du maître, telles que nous les avons analysées, permettent de dégager les principales caractéristiques de cette instance. Alvaro est celui qui dit NON. Non au départ pour le Nouveau Monde. Non à lévangélisation des Indiens d'Amérique. Non à la culture de l'exploitation et du profit. Non à la subordination de l'Ordre à l'autorité du Roi. Non à l'amour. Non au mariage. Non à la famille. Il ne dit oui qu'au retrait dans un couvent. Même s'il est docte, il ne s'inscrit pas dans la logique du passeur de savoir-vivre et de savoir-faire (voir Bonnet dans Noacco, 2013). Tranchant dans ses positions, il est plutôt dans le refus d'échanger et de transmettre. Cette intransigeance présente un côté agaçant. Montherlant a pris soin de ne pas présenter son personnage sous un jour mélioratif. Toutefois, on peut déceler à travers tout ce que celui-ci rejette l'affirmation indirecte de ce qu'il prône, donc de ce qu'il souhaite transmettre : la remise en question d'un système de valeurs fondé sur le culte du gain et de la richesse, la dénonciation d'une société phagocytée par le matérialisme barbare. Figure magistrale, Dieu est surtout présent dans le discours des personnages en tant que force dotée de deux attributs : la volonté, qui intervient dans la gestion de l'univers et du comportement de lêtre humain, et la grâce, qui est un processus d'élection utilisé pour sélectionner les meilleurs parmi l'ensemble des disciples. Le Roi, quant à lui, est un maître contesté, pour de multiples raisons : il est un usurpateur de pouvoir (il s'est autoproclamé Grand Maître des Ordres d'Espagne), étranger, trop jeune et mauvais gestionnaire des affaires de l'État. L'auteur lui, tire sa magistralité de son statut d'écrivain, donc de créateur, et de son engagement en tant qu'intellectuel : dénonciation des ravages de la colonisation.

Comment les disciples se situent-ils par rapport à ces figures?

\section{Figures du disciple}

Bien qu'il dise considérer les cinq chevaliers présents dans la pièce comme ses " pairs » (p. 32) et, parmi eux, Bernal comme un ami, ceux-ci sont conscients qu'ils ne sont pour lui que des disciples, chose qui d'ailleurs a fini par exaspérer le père de Jacinto : «Ah! Je suis fatigué de vous entendre nous donner des leçons» (p. 84). À cet ensemble s'ajoute Mariana, bien que son père l'ait toujours négligée et qu'il ne l'ait adoptée qu'après avoir su qu'elle lui a évité de commettre une grave erreur. Les cinq chevaliers constituent deux sous-groupes, celui des plus âgés qu'Alvaro (Bernal, cinquante-deux ans, Olmeda, soixante-deux ans, et Vargas, cinquante ans) et celui des plus jeunes (Obregon, trente-cinq ans et Letamendi, dix-neuf ans). On pourrait également les classer en deux catégories. La première réunit ceux qui ont participé à la bataille décisive de Grenade (Alvaro, Bernal et Olmeda) et la deuxième - les autres. Les informations fournies au sujet de ces personnages sont d'une importance inégale. Bernal a connu la paupérisation : " Notre maison a toujours décliné » (p. 73); résultat : «le seul héritage [...] que j’ai reçu de mes parents est l'honneur » (p. 72-73). Son principe est de « s'adapter ", d' « être vertueux dans le siècle » 
(p. 49-50). S'il ne part pas pour le Nouveau Monde, c'est uniquement pour des raisons de santé (voir p. 27, 38 et 73). Olmeda ne semble pas intéressé par les questions matérielles. S'il est candidat au départ pour le Nouveau Monde, c'est pour des considérations religieuses : "La nouvelle croisade est là » (p. 39). L'évangélisation des autochtones, il l'assimile à une œuvre de salut : « des millions d'Indiens brûleraient pour l'éternité en enfer, si les Espagnols ne leur apportaient pas la foi » (p. 45). Candidat lui aussi au départ, Obregon est peu disert sur sa personne. Ses rares interventions se limitent à la signification qu'il donne à ce départ et à l'attitude à adopter quand on choisit le désengagement. Il assimile la conquête du Nouveau Monde à un devoir qui incombe à ceux qui ont libéré l'Espagne (p. 34). Par ailleurs, selon lui, faire le choix de l'abstention implique une exigence morale, celle de ne pas se permettre de critiquer : "il y a plus délégance, quand on se retire du monde, de s'en retirer sans le blâmer » (p. 51). Inapte, Vargas partirait aux Indes "n’était [sa] misérable blessure » (p. 38). Ayant presque le même âge qu'Alvaro, il défend le principe de l'engagement total : « Ne serait-ce pas héroïsme [...] de jouer son rôle dans une société qui vous heurte, pour y faire vaincre [ses] idées? » (p. 49). La grâce divine s'acquiert par le respect de cet engagement : "il n'y a qu'une seule patrie, celle que formeront les Élus » (p. 48). Compte tenu de son jeune âge, Letamendi, que paradoxalement Alvaro pousse à partir pour subir des épreuves initiatrices, réduit cet acte à un exutoire : "On étouffe à Avila... » (p. 41), « je ne resterai pas dans cette ville effroyable, ce tombeau des tombeaux» (p. 56).

À cause de sa relation biologique avec le maître et de sa proximité avec lui, Mariana est, parmi les disciples, celle qui fait l'objet d'une focalisation. Jeune, belle, sensible mais " fière comme un aspic " (p. 97) alors qu'elle vit dans le dénuement total, elle tient beaucoup d'Alvaro. Elle a étudié les livres et l'histoire. Amoureuse, elle souhaiterait épouser Jacinto, mais paradoxalement elle confère au mariage une fonction peu courante. Elle répète : « je ne cherche pas à être heureuse » (p. 94), « je ne veux pas être heureuse » (p. 101). Son objectif, elle le place à un autre niveau : « je ne voudrais pas une vie facile. Je voudrais une vie où l'on aurait besoin de courage» (p. 95). De ce fait, elle envisage son rapport à l'argent d'une manière différente : la richesse, « je l'accueillerai comme une épreuve et je m'efforcerai de la surmonter » (p. 96).

Les figures analysées peuvent être classées en cinq catégories. La première, celle de la figure dite « aristotélicienne» (voir Noacco, 2013), regroupe Bernal et Vargas. Ils commentent et interprètent le discours du maitre. Celle de la figure dite « épicurienne » est incarnée par Olmeda : atteindre le bonheur est son objectif principal. Celle du disciple considéré comme "parricide " est représentée par Obregon, qui souhaite faire disparaitre Alvaro de la scène. À cause de ses maladresses, Letamendi est la figure du mauvais élève. Quant à Mariana, elle illustre bien la figure du disciple « alter ego » qui ressemble beaucoup au maître mais qui n'en est pas la réplique stérile.

La multiplicité des figures magistrales et des catégories de disciples nous invite à nous interroger sur les relations qui s'établissent entre les uns et les autres et sur les valeurs affirmées en vue dêtre inculquées. 


\section{Les relations maître-disciple et les valeurs affirmées}

Alvaro offre à ses "pairs " " une hospitalité [...] austère » (p. 19) qui ne favorise pas la communication, qui les fait fuir : « un [...] froid [...] se glisse en l'homme quand il se désaffectionne de quelque chose [...] l'Ordre de Santiago déchoit» (p. 18). La transmission semble être en panne, et pourtant ce sont ces difficultés qui en alimentent la dynamique. La persistance du maître à s'opposer au départ pour le Nouveau Monde et l'insistance de ses " compagnons » sur la nécessité d'y participer confèrent une vivacité à l'échange. D'un côté fusent les accusations, l'expression du mépris, la pédagogie de la comparaison, les paradoxes et léloge de l'épreuve. Alvaro pointe du doigt les agissements des membres de l'Ordre : "pleins d'indifférence ou d'indulgence pour l'ignoble, vous pactisez avec lui, vous vous faites ses complices!" (p. 52). Letamendi a rapidement saisi ce que le maître affiche à son égard : "Vous me dédaignez!» (p. 56). Vis-à-vis de Bernal, Alvaro use de la pédagogie de la comparaison en lui montrant comment il se comporte avec sa fille : "peut-être est-il bon que vous sachiez comme certains pères croient qu'ils doivent traiter leurs enfants " (p. 84). Il emploie le procédé du paradoxe dans le but d'étonner, d'amener à réfléchir : " Dans une guerre de cette espèce, la cause qui est sainte, c'est la cause des indigènes » (p. 42). Il préconise la souffrance comme épreuve qualifiante : «Quand vous hésitez entre plusieurs voies, prenez toujours la plus douloureuse » (p. 87). De l'autre côté, émergent les positions des disciples. Elles oscillent entre l'effet produit par l'intransigeance du maître, les critiques adressées à celui-ci et les principes réaffirmés. Le détenteur de l'autorité pratique la déstabilisation afin de désarçonner ses interlocuteurs et de faire prévaloir son évaluation des choses. Il choisit bien ses cibles : Letamendi est le maillon faible du groupe parce que le plus jeune et sans expérience, Olmeda est vulnérable parce qu'il est le plus âgé. Il décontenance le premier d'une manière progressive : il sème chez lui le doute ("Je me demande si je dois partir », p. 56), puis l'appréhension (« le cœur incertain et inquiet », p. 56), puis la perte de la sérénité («je suis troublé », p. 55) et, enfin, l'abattement («Ah ! vous me désespérez!», p. 56). Il va même jusqu’à lui dévoiler sa stratégie : «C'est ce que je veux » (p. 56). Olmeda, qui n’a pas échappé à cette épreuve, reconnaît l'efficacité de la démarche : "Le plus jeune et le plus vieux d'entre nous, vous les avez ébranlés » (p. 57). C’est sans doute cette "cruauté » (p. 75) qui a suscité la multiplicité des reproches adressés à Alvaro. On critique ses excès : "Vous n'avez pas cinquante ans, et vous parlez comme si vous en aviez quatre-vingts» (p.42). On fustige sa subjectivité : « "vous rejetez une époque faute de la voir comme elle est » (p. 49). On met à l'index sa passivité : «Debout sur le seuil de l'ère nouvelle, vous refusez d'entrer » (p. 49). La réaffirmation de la différence est la conséquence de cette attitude. À la démission et au retrait est opposée l'action : «l'ordre [...] est engagé là-bas dans une guerre sainte » (p. 42). La pièce ne nous dit cependant pas si les candidats au départ ont bravé ce qu'on pourrait appeler l'interdit. Elle s'est concentrée sur le rapport entre Alvaro et Mariana. Avant que n’ait lieu le changement total à la fin de l'œuvre, le père a toujours réitéré le manque 
d'affection qu'il éprouve pour sa fille et, par conséquent, l'absence de communication entre elle et lui. Sa proximité est vécue comme une charge génératrice d'agacement. Il la traite avec mépris. Cette distance a influé sur la relation didactique forcément présente ici. La responsabilité de l'éducation des enfants incombe d'abord aux parents. La mère de Mariana étant décédée, cette tâche relève des obligations d'Alvaro. Sa pédagogie présente quatre caractéristiques essentielles. La première consiste à recourir à l'exemple édifiant : "Comme les Spartiates montraient à leurs fils un ilote ivre, il arrive que je lui montre mon pays, pour qu'elle voie ce qu'elle ne doit pas être » (p. 68). La seconde fait appel à la déduction : « je lui ai appris [...] un peu d'histoire : elle saura comment les empires meurent » (p. 68). La troisième s'appuie sur le procédé de la dépréciation des valeurs autres que celles qu’on veut transmettre : "Vous êtes une petite singesse, rien de plus» (p. 86). La quatrième pousse plus loin la manœuvre de la subordination en utilisant l'accusation : "c'est vous ma pierre d'achoppement " (p. 87). Mais Mariana a toujours été une " élève " aimante et respectueuse. L’enjeu de ces relations à formes multiples se situe au niveau des valeurs affirmées afin dêtre inculquées.

En rejetant tout ce qui est communément admis, Alvaro vise malgré tout à transmettre avant de disparaitre. Dans les principes qu'il a adoptés, nous relevons essentiellement six idées-forces. Il s'agit d'une certaine conception de l'histoire, d'une définition du rôle de la chevalerie et des quatre conditions à remplir pour être digne de respect. L'histoire nous procure un enseignement précieux. Elle nous apprend qu'il faut être toujours méfiant à l'égard des forces négatives qui sont derrière les événements : « les valeurs nobles, à la fin, sont toujours vaincues ; l'histoire est le récit de leurs défaites renouvelées » (p. 52). C’est par rapport à cette menace permanente qu'est fixé le rôle du chevalier : « la chevalerie est essentiellement la défense des persécutés » (p. 42). L'engagement de tout être dans la même voie requiert la possession de quatre qualités morales. L'affirmation de soi passe en premier lieu par l'autodéfense : « La gloire de l'Espagne a été de réduire un envahisseur dont la présence insultait sa foi, son âme, son esprit, ses coutumes » (p. 44). Elle exige en second lieu le sacrifice de soi : « la générosité, c'est toujours le sacrifice de soi ; il en est l'essence » (p. 127). Vient en troisième lieu l'importance de la charité : «les hommes n'existent que pour être objet de charité » (p. 112). La qualification pour ces acquis nécessite un travail sur soi, un passage par l'épreuve de la souffrance : « lutte, souffre davantage. Où il n'y a pas de combat il n'y a pas de rédemption » (p. 127).

Si Montherlant ne partage pas l'extrémisme d'Alvaro, il le rejoint, en tant que maitre lui aussi, au sujet de la condamnation de la colonisation, cette pratique qui consiste à évincer une civilisation pour la remplacer par une autre. C'est sans doute la principale leçon qu'on puisse tirer de la relation didactique inscrite dans la pièce. 


\section{RÉFÉRENCES}

Deshoulières, V. et Constantinescu, M. (2009). Les Funambules de l'affection. ClermontFerrand : Presses Universitaires Blaise Pascal.

Franck, J. (2003). Maître et disciple ; quel lien ? Récupéré de https://www.lalibre.be>culture>livres-bd

Kieffer, M. (2013). Maître et disciple : les relais du savoir des premières théories à nos jours. Acta Fabula, vol. 4, n 6. Récupéré de http://www.fabula.org/acta/document8047.php

Montherlant, H. (1991). Le Maître de Santiago [1947]. Paris : Gallimard.

Noacco, C., Bonnet, C., Marot, P. et Orfanos C. (2013). Figures du maître. De l'autorité à l'autonomie. Rennes : Presses Universitaires de Rennes.

Scheler, M. (2003). Nature et formes de la sympathie. Contribution à l'étude des lois affectives. Paris : Payot.

Steiner, G. (2003). Maîtres et disciples. Paris : Gallimard.

Vissac, T. (2012). Maîtres et disciples selon Mariana Caplan. Récupéré de www.istenqs.org/ Caplan_gourou.htm

Wolff, F. (2006). L'être, l'homme, le disciple. Figures philosophiques empruntées aux anciens. Paris : Presses Universitaires de France.

RÉSUMÉ : La question du rapport maître-disciple est mise en évidence dans Le Maître de Santiago de Montherlant par une théâtralisation de la transmission. Dialogue vif et tendu, situations conflictuelles et divergences de points de vue ébranlent aussi bien les relations familiales et amicales que les affinités idéologiques. Pour les Espagnols de 1519 partir en Amérique est une aubaine. Cette course au gain effrénée qui utilise l'évangélisation comme prétexte séduit les chevaliers de l'Ordre de Santiago mais elle déplaît énormément à leur chef. La focalisation de cette dramatisation sur le différend en question permet de dégager les figures du maître, celles du disciple et, à travers leurs relations, les valeurs affirmées. Alvaro Dabo, qui agit en tant que guide de l'Ordre de Santiago, est sans conteste la principale figure du maître. En cultivant le paradoxe et en s'opposant au désir de ses pairs, il ne s'érige pas en passeur de savoir-vivre et de savoir-faire, mais il parvient tout de même à formuler les valeurs auxquelles il est attaché : noblesse d'âme, générosité, humilité. Les disciples se répartissent en cinq catégories. La figure « aristotélicienne » (Bernal et Vargas) commente et interprète le discours du maître. La figure " épicurienne » (Olmeda) vise à atteindre le bonheur. Le disciple " parricide » souhaite éliminer Alvaro. Letamendi est l'illustration du mauvais élève. Mariana, incarnation de l' " alter ego ", tient du maître sans en être une pâle copie. Tendues, les relations qui régissent les deux instances confèrent une dynamique au processus de transmission. Alvaro cherche à se murer dans le silence, mais il tient à transmettre avant de disparaître. Deux valeurs essentielles constituent la teneur de son message : l'histoire nous enseigne qu'il faut être méfiant à l'égard des forces négatives, l’engagement requiert des qualités morales 
telles que le sacrifice de soi et la charité. Montherlant approuve chez son personnage la condamnation de la colonisation, mais il n’adhère pas à son extrémisme.

Mots-clés : Henry de Montherlant, transmission, conflit, figures magistrales, engagement, retrait

\section{Dramatization of the transmission in Le Maître de Santiago by Montherlant} ABSTRACT: The question of the master-disciple relationship is highlighted in Le Maitre de Santiago (The Master of Santiago) of Montherlant by a theatricalization of the transmission. Lively and tense dialogue, conflicting situations and divergent points of view undermine family and friend relations as well as ideological affinities. For the 1519 Spaniards the chance to go to America is a golden opportunity. This frantic race behind the gain that uses evangelism as a pretext, seduces the knights of the Order of Santiago, but greatly displeases their leader. The focus of this dramatization on the dispute in question allows us to identify the figures of masters, those of disciples and, through their relations, the affirmed values. Alvaro Dabo, who acts as a guide to the Order of Santiago, is undoubtedly the main figure of master. By cultivating the paradox and opposing the desire of his peers, he does not set himself up as a transmitter of good-manners and of know-how, but he nevertheless manages to formulate the values to which he is attached: nobility of soul, generosity, humility. The disciples are divided into five categories. The "Aristotelian" figure (Bernal and Vargas) comments and interprets the master's speech. The "Epicurean" figure (Olmeda) aims to achieve happiness. The parricide disciple wants to eliminate Alvaro. Letamendi is the embodiment of a bad student. Mariana, the incarnation of the master's "alter ego", looks like the master without being his pale copy. Being tense, the relations that govern the two instances give a dynamic to the process of transmission. Alvaro tries to confine himself to silence, but before disappearing he wants to transmit his message. Two essential values constitute its content: history teaches us that one must be distrustful of negative forces; commitment requires moral qualities such as self-sacrifice and charity. Montherlant approves of his character's condemnation of colonization but he does not adhere to his extremism.

Keywords: Henry de Montherlant, transmission, conflict, master figures, engagement, withdrawal 